\title{
Analysis of skeletal development on the basis of body mass and bone resorption
}

\author{
G.L. Wright ${ }^{\mathrm{a} *}$, D.L. DeMoss ${ }^{\mathrm{b}}$, W. Geng ${ }^{\mathrm{a}}$ \\ "Department of Physiology: Marshall University School of Medicine, 1542 Spring Valley Drive. \\ Huntington, WV 25704, USA \\ ${ }^{b}$ Department of Biological and Environmental Sciences, Morehead State University, Morehead, KY 40351, USA
}

\begin{abstract}
Regression analysis was utilized to define the relationships among body weight, whole skeleton bone resorption ( ${ }^{3} \mathrm{H}$-tetracycline method), and the development of skeletal mass. The results indicated that skeletal development ( $\%$ body weight) of slowly growing, 24-week-old rats consisted of two major components, one directly $(r=0.985, P<0.001)$ and one inversely $(r=0.977, P<0.001)$ related to body weight. It was further noted that the skeletal resorption rate was inversely correlated to body weight $(r=0.879 . P<0.05)$ and directly related to skeletal development $(r=0.865, P<0.05)$, suggesting that whole skeleton bone resorption and formation were highly correlated in the slowly growing animal. A third small component of skeletal development identified in the analysis of data from 24week-old animals, showed no direct relationship to either body weight or resorptive activity. The model presented enables the separation of skeleton mass into major components which may represent mechanically and metabolically driven bone formation. (c) 2000 Elsevier Science Inc. All rights reserved.
\end{abstract}

Keywords: Skeleton mass; Bone resorption; Body weight; Mechanical bone formation; Metabolic bone formation

\section{Introduction}

In a recent report (1), the whole skeleton mass of male and female rats of the SpragueDawley (SD), Wistar Kyoto (WKy), and spontaneously hypertensive (SHR) rat strains was determined. From this preliminary analysis a clear relationship between body weight and skeletal mass was observed. The analysis of these data have been extended with the surprising finding of similar relationships between body weight and skeleton mass in each of 4,8 , 16 , and 24 week age groups of rats. At each age, approximately $2.2 \mathrm{~g}$ of skeletal mass per $100 \mathrm{~g}$ of body weight present in the rat was directly correlated to increased body weight, suggesting that the relationship between these parameters is constant across rat strain, gender,

* Corresponding author. Tel.: (304)-696-7368; fax: (304)-696-7381.

E-mail addresses: wrightg@marshall.edu (G.L. Wright) 
and growth phase. A second component of skeletal mass that was independent of body weight was increased with age. In the present report, we have examined in greater detail the relationships among body weight (BW), whole skeleton bone resorption (R), and skeletal mass (SM) in 24 week old rats. The results indicate that skeletal development (Sd $=\left(S_{1} M\right)$ $\mathrm{BW}(100)$ ) may be resolved into two major components. One component is directly correlated with body weight and may reflect skeletal bone formed and maintained in response to mechanical support demands. A second component is inversely related to Sd but is directly correlated with the rate of bone resorption, suggesting a portion of skeletal development potentially related to skeletal remodeling and body calcium homeostasis. Some of the data utilized in the present work has been published elsewhere $(1,2)$.

\section{Methods}

\section{Animals}

The experiments performed in the study were approved by the Institutional Animal Care and Use Committee. All rats were maintained in colony rooms at $23 \pm 2{ }^{\circ} \mathrm{C}$ on a 12 -h light/ dark photoperiod. Animals received free access to Purina Rat Chow and tap water. The strains of rats utilized were Sprague-Dawley (SD/Hla BR. Hilltop Lab Animals. Scottsdale, PA), Wistar Kyoto (WKy/NCrlBR, Charles River, Wilmington, MA), and Aoki-Okamoto spontaneously hypertensive rats (SHR/NCrlBR) Charles River, Wilmington, MA).

\section{Dry skeletal mass determination}

A total of 12 Sprague-Dawley (SD), 12 Wistar Kyoto (WKy), and 12 spontaneously hypertensive rats (SHR), including both sexes at 24 weeks of age were sacrificed for determination of body weight and dry skeletal mass. Animals were euthanized by $\mathrm{CO}_{2}$ inhalation and the body weight immediately determined. The carcasses were then stripped of as much soft tissue as possible prior to being placed on mesh trays in chambers containing Dermestid beetles (Dermestes maculatus, Carolina Biological Supply Co., Burlington, NC). The polished skeletons were cleaned by an ethyl alcohol soak followed with repeated rinses in distilled water. The cleaned skeletons were defatted by submerging each skeleton in $200 \mathrm{ml}$ of $100 \%$ ethanol with the ethanol changed a minimum of three times during the two week interval in which the bones were extracted. Following the extraction, the skeletons were lyophilized to constant weight.

\section{Measurement of whole skeleton bone resorption}

In a separate experiment, a modified ${ }^{3} \mathrm{H}$-tetracycline protocol (2) was employed to measure whole skeleton bone resorption in the different rat strains. Thirteen days prior to the initiation of urine collection for ${ }^{3} \mathrm{H}$-tetracycline (NET-141 Tetracycline, $\left[7-{ }^{3} \mathrm{H}(\mathrm{N})\right]-$; New England Nuclear, Boston, MA) determination, groups of male and female rats $(\mathrm{N}=6)$ of each rat strain (SD, WKy, SHR) at 22 weeks of age underwent a series of subcutaneous injections of ${ }^{3} \mathrm{H}$-tetracycline dissolved in $0.05 \mathrm{M} \mathrm{HCl}$ containing ascorbic acid. At the conclusion of the labeling period, each of the rats received $35 \mu \mathrm{Ci} /$ rat in five injections on days $1,4,7,11$ and 14 of the labeling interval. Previous work (2) has shown that the kinetic profile of urinary
${ }^{3} \mathrm{H}$-tetracycline loss .animals. Their resul tbolically reactive $s i$ icalcium diets. Foll metabolic cage and Idaily. The first $24-\mathrm{r}$ for a period of $35 \dot{c}$ Scintiverse E (Fish by liquid scintillatic skeletal mass at the Curve Fitting Softy curve best fit equat: the $y$-intercept of th labeled. This param tion in subsequent $c$

\section{Results}

Fig. 1 shows re: body weight taken.

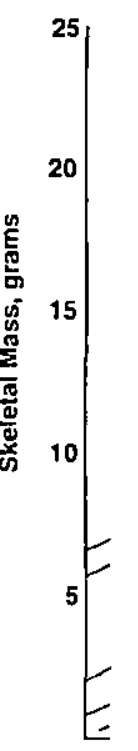

Fig. 1. Relationship be Kyoto (WKy) and spor from whole skeleton $\mathrm{d}$ Symbols indicate grour at $2.2 \%$ of body weight 
It of body : detail the id skeletal id $=(\mathrm{SM} /$ ctly correesponse to is directly pment poe data uti-

imal Care 12-h light/ vater. The icottsdale, -Okamoto

sly hyperrmination on and the soft tissue id beetles I skeletons vater. The sanol with the bones eight.

d to meato the ini$v$ England $f$ each rat ections of ion of the 7,11 and of urinary
${ }^{3} \mathrm{H}$-tetracycline loss of rats labeled for two weeks is identical to that of chronically labeled animals. Their results, therefore, indicate that the two week interval is adequate to label metabolically reactive sites of bone turnover in the skeletons of sedentary animals receiving $1 \%$ calcium diets. Following the final isotope injection, each rat was transferred to a standard metabolic cage and its food consumption, body weight, and urine volume were determined daily. The first 24-h urine was discarded and urine was thereafter collected at 24 -h intervals for a period of 35 days. A $0.5 \mathrm{ml}$ aliquot of each urine sample was combined with $10 \mathrm{ml}$ of Scintiverse E (Fisher Scientific, Pittsburgh, PA) and the ${ }^{3} \mathrm{H}$-tetracycline content determined by liquid scintillation spectrometry. The urinary ${ }^{3} \mathrm{H}$-tetracycline data were normalized for dry skeletal mass at the end of isotope injections (1) and analyzed via Table Curve 2D Automated Curve Fitting Software (Jandel Scientific, San Rafael, CA) to determine the ${ }^{3} \mathrm{H}$-tetracycline curve best fit equation. The ${ }^{3} \mathrm{H}$-tetracycline urinary loss curves were then analyzed to obtain the y-intercept of the slow component, the rate of label loss at time zero with the pool fully labeled. This parameter was then used as the measure of rate of whole skeleton bone resorption in subsequent calculations.

\section{Results}

Fig. 1 shows regression analyses of the relationship between whole skeleton mass and body weight taken at different ages in rats from three strains showing significant differences

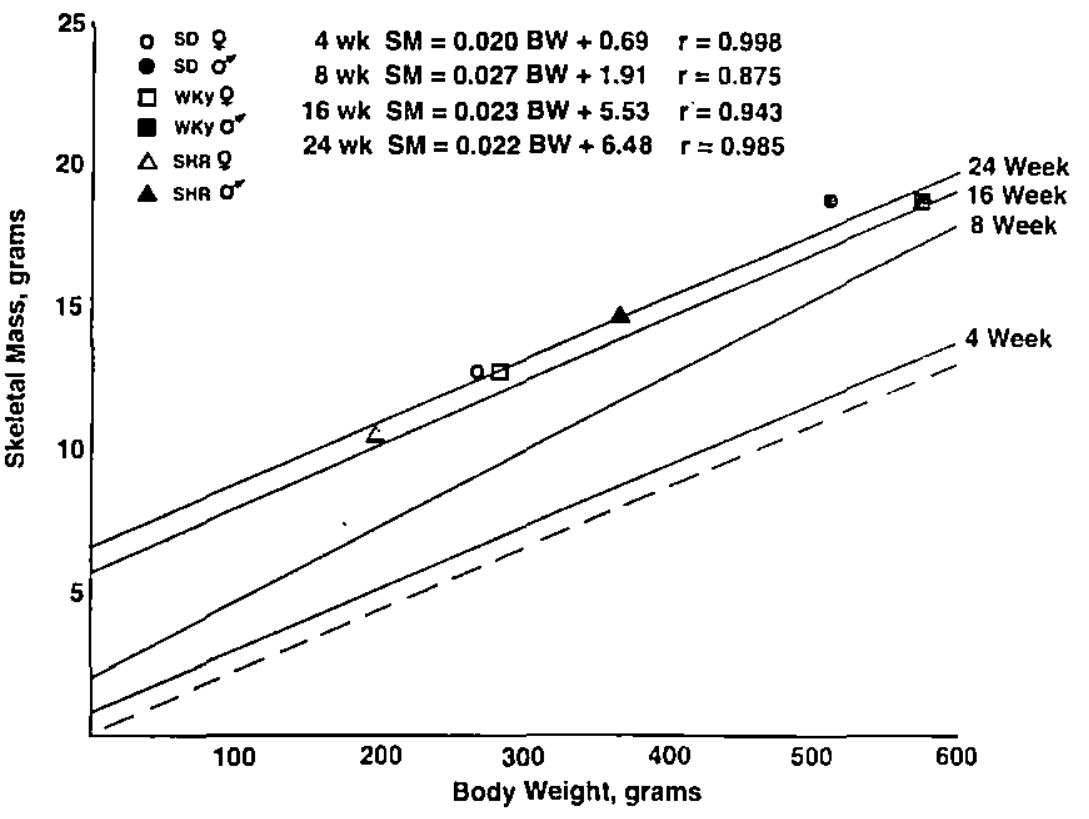

Fig. 1. Relationship between skeletal mass and body weight in male and female Sprague-Dawley (SD). Wistar Kyoto (WKy) and spontaneously hypertensive (SHR) rats. Lines were drawn from regression equations derived from whole skeleton dry weight vs body weight of the six groups of animals at 4. 8. 16. and 24 weeks of age. Symbols indicate group averages $(n=6)$ for the rats at 24 weeks of age. The dashed line indicates skeletal mass at $2.2 \%$ of body weight. Data taken from DeMoss and Wright. 1998. 
in genetically determined body growth (data from DeMoss and Wright, 1998). The data suggest that at each age between 4 and 24 weeks, the skeletal mass present in the animal consisted of two components; (i) a component proportionately related to increasing body weight at about $2.2 \%$ of body weight, and; (ii) a second component of mass that was independent of increasing body weight. These results, therefore suggest that the portion of growth and maintenance of skeletal mass directly correlated with increasing body weight is constant across age, sex, and rat strain and may be calculated at approximately $2.2 \%$ of body weight. The second component of skeletal mass increased with age and is the cause of the increase in the ratio of skeletal mass to body weight with aging and small body size previously observed (1).

We have suggested that the portion of the skeletal mass developed independently of increasing body weight is related to body calcium homeostasis and may be independent of bone formed in direct response to mechanical strain (1). In the present analysis, the relationship between body weight and bone metabolic parameters with skeleton mass was examined in 24-week-old animals expected to have little additional skeletal growth with predominately bone remodeling activity. The three rat strains chosen for study represent a relatively wide range in body weight and skeletal bone resorption values (Table 1). Because each group of animals represents a different genetically determined body size, the average value of each group was utilized for regression analysis.

\section{4-Week-old rats}

The relationship between dry skeletal mass (SM) and body weight (BW) of mature 24week-old rats (Fig. 2A) within the weight range of $200 \mathrm{~g}$ to $575 \mathrm{~g}$ (equation a) indicates that only a portion of skeleton mass is directly correlated with increasing.body weight.

$$
S M=0.022 B W+6.48 \quad r=0.985, P<0.001
$$

The contribution of the second component formed independently of body weight to the relationship between skeletal mass and body weight in the 24-week-old rat is emphasizedi when

Table 1

Skeletal mass and metabolic resorption parameters for Sprague-Dawley (SD). Wistar Kyoto (WKy) and spontaneously hypertensive rats (SHR) at 24 weeks of age

\begin{tabular}{llccc}
\hline 24 Week & ${\text { Body wt, } \mathbf{g}^{\prime}}$ & Skeletal wt, $\mathrm{g}^{1}$ & Skel/body. \% & Bone resorption \\
\hline Male & & & & \\
SD & $509.1 \pm 28.7$ & $18.8 \pm 0.7$ & $3.7 \pm 0.1$ & $5.8 \pm 0.6$ \\
WKy & $578.0 \pm 28.4$ & $18.8 \pm 0.5$ & $3.3 \pm 0.1$ & $8.2 \pm 0.6$ \\
SHR & $362.4 \pm 12.5$ & $14.8 \pm 0.5$ & $4.1 \pm 0.1$ & $9.6 \pm 0.4$ \\
Female & & & & \\
SD & $261.4 \pm 10.8$ & $12.8 \pm 0.4$ & $5.0 \pm 0.2$ & $12.9 \pm 0.6$ \\
WKy & $277.1 \pm 6.1$ & $12.7 \pm 0.2$ & $4.6 \pm 0.1$ & $12.5 \pm 0.8$ \\
SHR & $196.4 \pm 3.7$ & $10.4 \pm 0.2$ & $5.3 \pm 0.1$ & $22.6 \pm 2.4$ \\
\hline
\end{tabular}

Values represent the mean \pm SEM of six animals.

'Taken from DeMoss and Wright, 1998.

* Urinary ${ }^{3} \mathrm{H}$-tetracycline loss, dpm/gm skeleton $\times 10^{3}$.
Fig. 2. The relationsl calculated as skeleton related with body we point represents the $g$

data are plotted a lated as percent $\left(\mathrm{Sd}_{\mathrm{T}}\right)$ in different weight (Fig. 2B)

$$
S d_{T}=
$$

Because it is $k$ with body weigh 1 
18). The data sug1 the animal consing body weight as independent of growth and main$s$ constant across sody weight. The he increase in the usly observed (1). ependently of in-? e independent of ysis, the relation-: ass was examined ith predominately a relatively wide sse each group of, age value of each

W) of mature 24$\mathrm{n}$ a) indicates that weight. y weight to the reemphasized when

(WKy) and

Bone resorption*

$5.8 \pm 0.6$

$8.2 \pm 0.6$

$9.6 \pm 0.4$

$12.9 \pm 0.6$

$12.5 \pm 0.8$

$22.6 \pm 2.4$
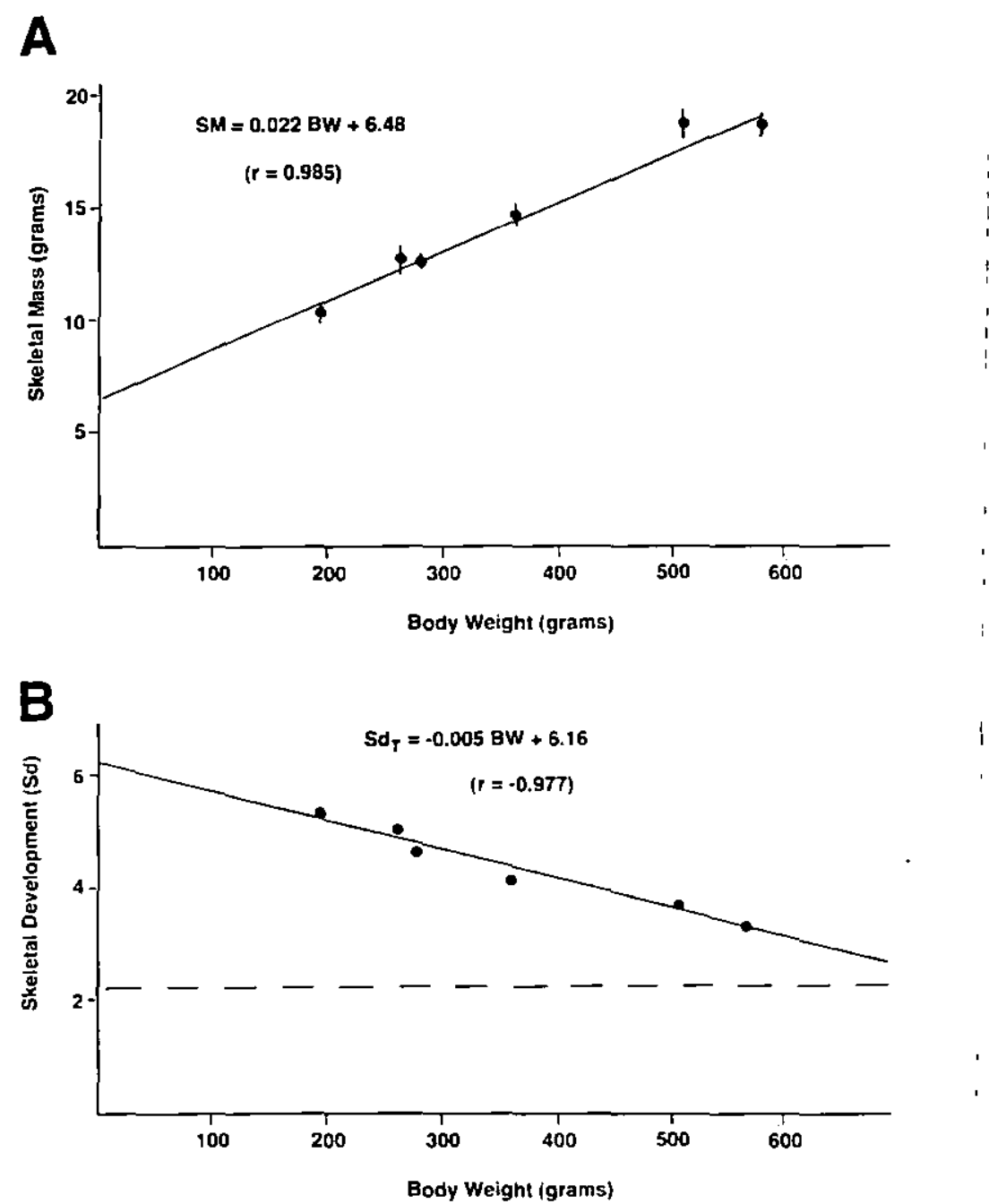

Fig. 2. The relationship between body weight (BW) and: $A)$ dry skeleton mass or, $B$ ) skeletal development (Sd) calculated as skeleton percent of body weight. The dashed line indicates the percent of skeleton mass directly correlated with body weight. Values were taken from 24-week-old, male and female SD, WKy and SHR rats. Each point represents the group average of 6 animals. Data taken from DeMoss and Wright, 1998.

data are plotted as a ratio of skeleton mass to body weight. Hence, if skeletal mass is calculated as percent of body weight to obtain a measure of relative total skeletal development $\left(\mathrm{Sd}_{\mathrm{T}}\right)$ in different sized animals, an inverse relationship is observed between $\mathrm{Sd}_{\mathrm{T}}$ and body weight (Fig. 2B) which is described by the equation:

$$
S d_{T}=\frac{S M}{B W} \cdot 100=6.16-0.005 B W \quad r=-0.977, P<0.001 .
$$

Because it is known from equation (a) that a constant $2.2 \%$ of $\mathrm{Sd}_{\mathrm{T}}$ is directly correlated with body weight, we may rewrite equation (b) to indicate that $\mathrm{Sd}_{\mathrm{T}}$ consists of two compo- 
nents; one (2.2\%) directly correlated to body weight, and a second component (3.96-0.005 $\mathrm{BW}$ ) inversely correlated to body weight.

$$
S d_{T}=2.2+(3.96-0.005 B W)
$$

Relationship between resorption, body weight, and skeleton mass

Fig. 3A shows that the measured tetracycline rate of whole skeletal bone resorption $(R)$ in mature 24-week-old rats (female SHR excluded) is inversely related to body weight as described by the equation:

$$
R=17.19-0.019 B W \quad r=-0.879, P<0.05
$$

\section{A}
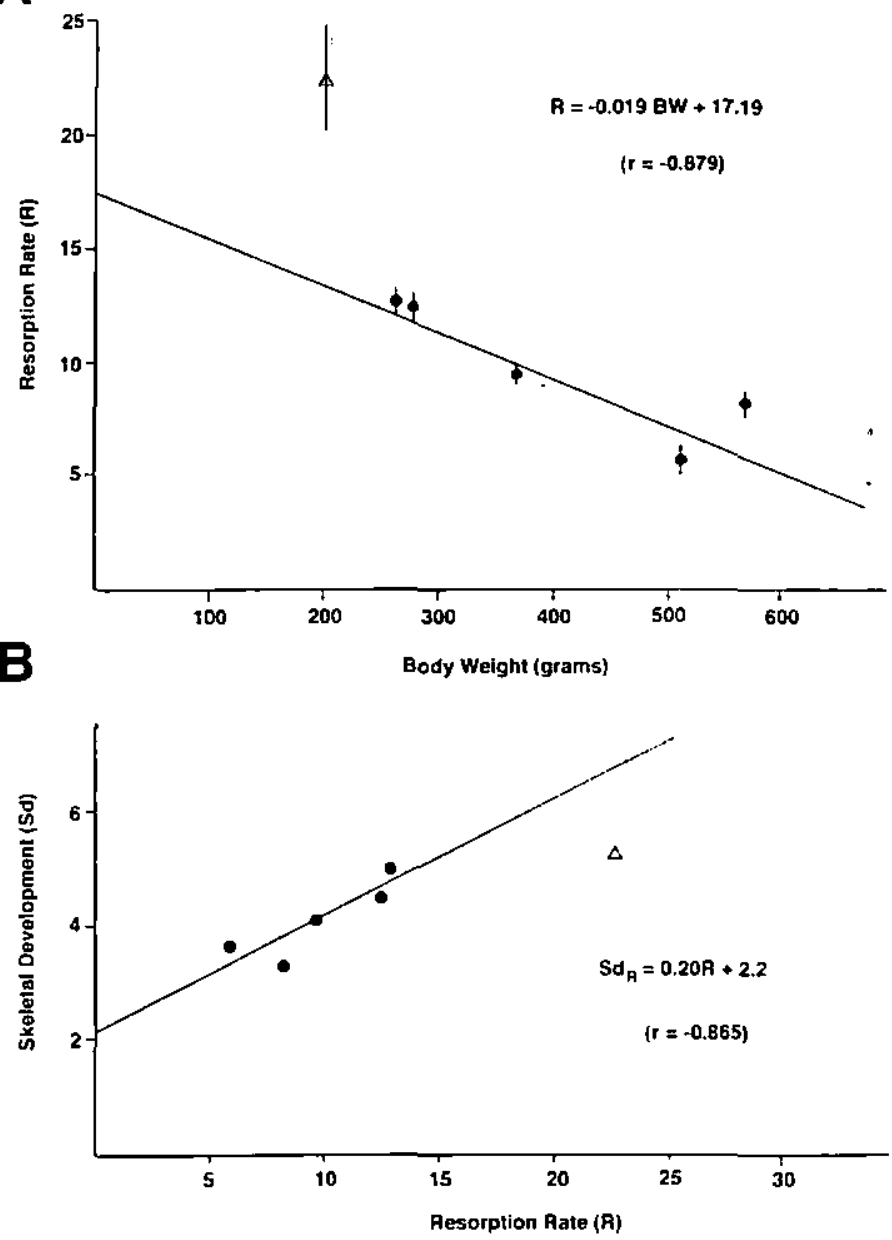

Fig. 3. The relationship between; A) whole skeleton bone resorption rate (R) and body weight (BW) and. B) skeletal development, $(\mathrm{SM} / \mathrm{BW})(100)$, and whole skeleton resorption rate. Data were taken from 24-week-old. male and female SD. WKy, and SHR rats. Note that female SHR $(\Delta)$ were not included in this analysis due to evidence of excessive resorptive activity. Each point represents the group average of 6 animals.
However, the skeletal develop

Hence, the an opment, the perc mass is compri weight, and a se

This equatior etal developme nents as a functi

This graph fu equal to $\mathrm{Sd}_{\mathrm{R}}$ (eq

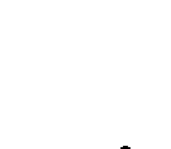

$$
\text { 8. }
$$$$
6-
$$$$
\text { व் }
$$

4 -

$2-$

Fig. 4. The relatiol three components resorptive activity , body weight or res: ( $\mathrm{Sd}_{\mathrm{T}}$; equation b) $0 \mathrm{i}$ of animals from wh 
e resorption $(\mathrm{R})$ in ıdy weight as de-

(c)
However, the rate of bone resorption can be demonstrated to be directly correlated with skeletal development (Fig. 3B) as described by equation:

$$
S d_{R}=\frac{S M}{B W} \cdot 100=0.25 R+2.2 \quad r=0.865, P<0.05
$$

Hence, the analysis of the relationship between whole skeleton resorption and skeletal development, the percent of body weight attributable to skeletal dry mass, also indicates that skeleton mass is comprised of two components. One component may be calculated at $2.2 \%$ body weight, and a second component that is directly correlated with bone resorption activity $(0.2 \mathrm{R})$.

This equation may be further developed to indicate the relationship of this portion of skeletal development to body weight allowing a plot of Sd to indicate the contributing components as a function of body weight (Fig. 4). From equations (c) and (d):

$$
\begin{aligned}
& S d_{R}=0.2(17.19-0.19) B W+2.2 \\
& S d_{R}=(3.44-0.0038 B W)+2.2
\end{aligned}
$$

This graph further indicates that $\mathrm{Sd}_{\mathrm{T}}$ (equation $\mathrm{b}$ ), derived from measured $\mathrm{Sd}$ data, was not equal to $\mathrm{Sd}_{\mathrm{R}}$ (equation e) derived from resorption data such that:

$$
\begin{gathered}
S d_{T}-S d_{R}=(6.16-0.005 B W)-(5.64-0.0038 B W) \\
S d_{T}-S d_{R}=0.52-0.0012 B W
\end{gathered}
$$

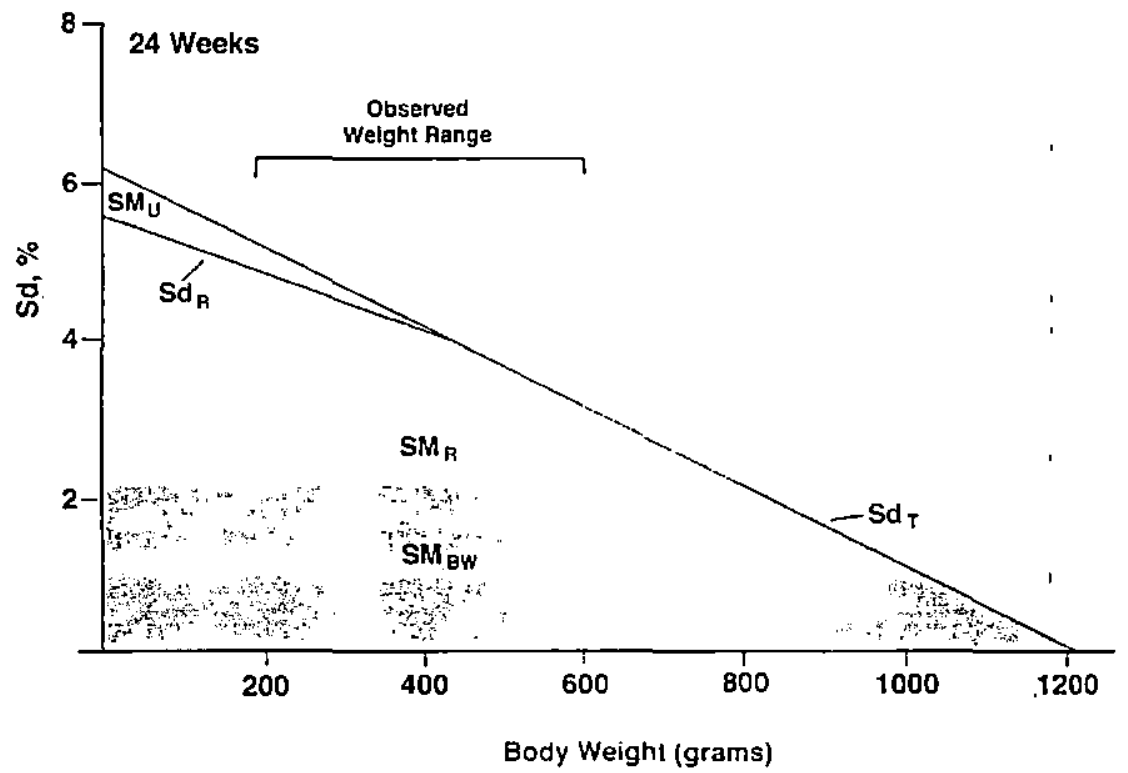

Fig. 4. The relationship between skeletal development and body weight in 24-week-old rats. The figure indicates three components of $\mathrm{Sd}$; that directly correlated to body weight $\left(\mathrm{SM}_{\mathrm{Bw}}\right)$. that calculated from whole skeleton resorptive activity $\left(\mathrm{SM}_{\mathrm{R}}\right)$, and a third component of skeletill mass nol found to be directly correlated with either body weight or resorption $\left(S_{U}\right)$. Lines were drawn from equations relating skeletal development to body weight $\left(\mathrm{Sd}_{\mathrm{T}}\right.$; equation $\left.\mathrm{b}\right)$ or to resorption rate $\left(\mathrm{Sd}_{\mathrm{R}}\right.$ : equation $\left.\mathrm{e}\right)$. Brackets indicate the range of body weights of the groups of animals from which data were derived. 
This difference may represent imprecision in the equations for $\mathrm{SD}_{\mathrm{T}}$ and $\mathrm{SD}_{\mathrm{R}}$ resulting from variability in the biological data from which they are derived. Alternatively, the difference could reflect the presence of a third small component of skeletal development in the 24 week old rat that is inversely correlated with body weight but that is not correlated with whole body bone resorption. The results suggest that the skeletal mass formed and then maintained in mature rats, is in one part directly related to increasing body size (equation a) which may be dominated by mechanisms associated with mechanical strain. A second large component is independent of body weight, is inversely related to skeletal development, is directly correlated with bone resorptive activity (equation d) and may represent a reservoir for calcium homeostasis and bone remodeling. In any case, the finding that a portion of skeletal development is highly correlated with resorptive activity suggests that whole skeletal net resorption and bone formation in this latter component of skeletal mass are tightly coupled in the mature animal, most likely reflecting predominantly remodeling activity. A third small component of skeletal bone formation (equation $\mathrm{f}$ ) appears to be inversely related with body weight, is not correlated with bone resorptive activity but would be of significance only in animals at body weights below $400 \mathrm{~g}$ (Fig. 4).

\section{Discussion}

In the past decade, Frost and collaborators (3-9) have advanced a series of concepts to explain the mechanism underlying bone shaping, size. and strength. They propose that these parameters are principally determined by two independently acting modes of osteoblast/ osteoclast activity. During global modeling, independent formation and resorption drifts shape bone and serve to increase bone mass. In comparison, remodeling by basic multicellular units (BMU) of osteoclasts and osteoblasts involves a highly coordinated and sequential resorption and reformation of bone at the resorption site. Remodeling maintains bone in good repair and may serve only to conserve or reduce bone mass. It is further proposed that both modes of activity are, in turn, activated or deactivated by a sensor mechanism responsive to mechanical strain. Hence, mechanically activated modeling or remodeling represent the primary initiating event which may. in turn, be influenced by nonmechanical humoral, dietary, and other factors. This concept of interdependent obligatory mechanical and permissive nonmechanical regulatory control of bone metabolism has formed an attractive hypothesis for explaining the apparent complexity of bone metabolism in the intact animal. Hence, there is presently great interest in the mechanism(s) by which bone cell function is modulated by mechanical stimuli. For example, it has been shown that immobilization and weightlessness reduce bone mass $(10,11)$ concurrent with decreased bone formation $(12,13)$ and increased bone resorption $(14,15)$. Conversely, increased mechanical strain may result in decreased resorption and increased bone formation (16-18). In a recent review of this topic, Rodan (19) concluded that the responsiveness of skeletal structure to mechanical force can only be explained in terms of: (a) an effective bone cell sensory mechanism for identification of bone matrix strain; (b) remodeling-mediated resorption of mechanically inactive bone, and; (c) unabated bone formation as long as mechanical strain is above threshold level. Hence, a feedback loop is proposed in which bone resorption and formation are coupled by the sensory mechanism for 1 bone formation

It has long $b$ cluding; the resf homeostasis (20 tionship betwee adult macaque $\mathrm{r}$ and osteon dens chanical and me abolically driver mals; whereas, $\mathrm{t}$ growing animal. lism of vertebra young growing :

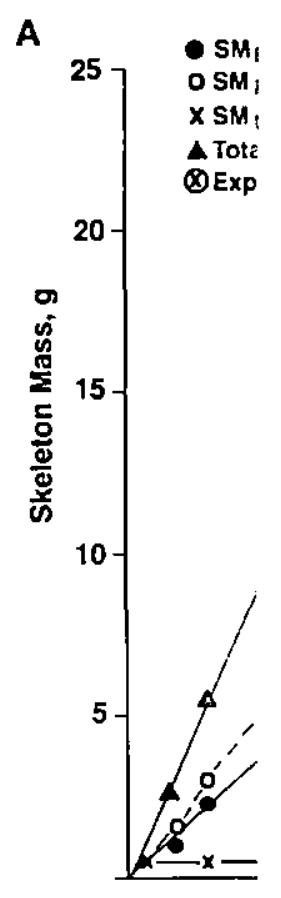

Fig. 5. Values calc weight (B) at a bor mass directly corre body weight and $d$ not directly correla individual compon DeMoss and Wrig] 
$S D_{R}$ resulting rely, the differment in the 24 zorrelated with rmed and then ze (equation a) A second large lopment, is dia reservoir for tion of skeletal skeletal net reitly coupled in A third small ated with body nce only in an-

:oncepts to exrose that these of osteoblast/ sorption drifts sic multicelluand sequential $s$ bone in good osed that both 1 responsive to resent the prinoral, dietary, srmissive nonnypothesis for Ience, there is lulated by meshtlessness reand increased decreased rec, Rodan (19) in only be exation of bone e, and; (c) unIence, a feed'y the sensory mechanism for detection of mechanical strain; resorption increases strain eliciting increased bone formation to reduce strain.

It has long been accepted that bone metabolic activity reflects a number of functions including; the response to mechanical strain, repair of fatigue damage, and whole body calcium homeostasis (20). In a recent study, Bouvier and Hylander (21) reported no consistent relationship between recently formed osteons and peak strain areas of the facial skeleton of the adult macaque monkey. By comparison, a positive relationship between peak strain regions and osteon density was obtained in growing, immature animals. They concluded that mechanical and metabolic factors contributed equally to bone metabolism or, alternatively, metabolically driven bone metabolism occurred without influence from strain levels in adult animals; whereas, bone metabolism was dominated by the influence of mechanical factors in the growing animal. In a second study, Erben (22) has shown that the nature of mineral metabolism of vertebral and tibial cancellous bone changes with aging in rats. He found that, in young growing animals, a major portion of bone metabolism resulted from modeling or mini-

A
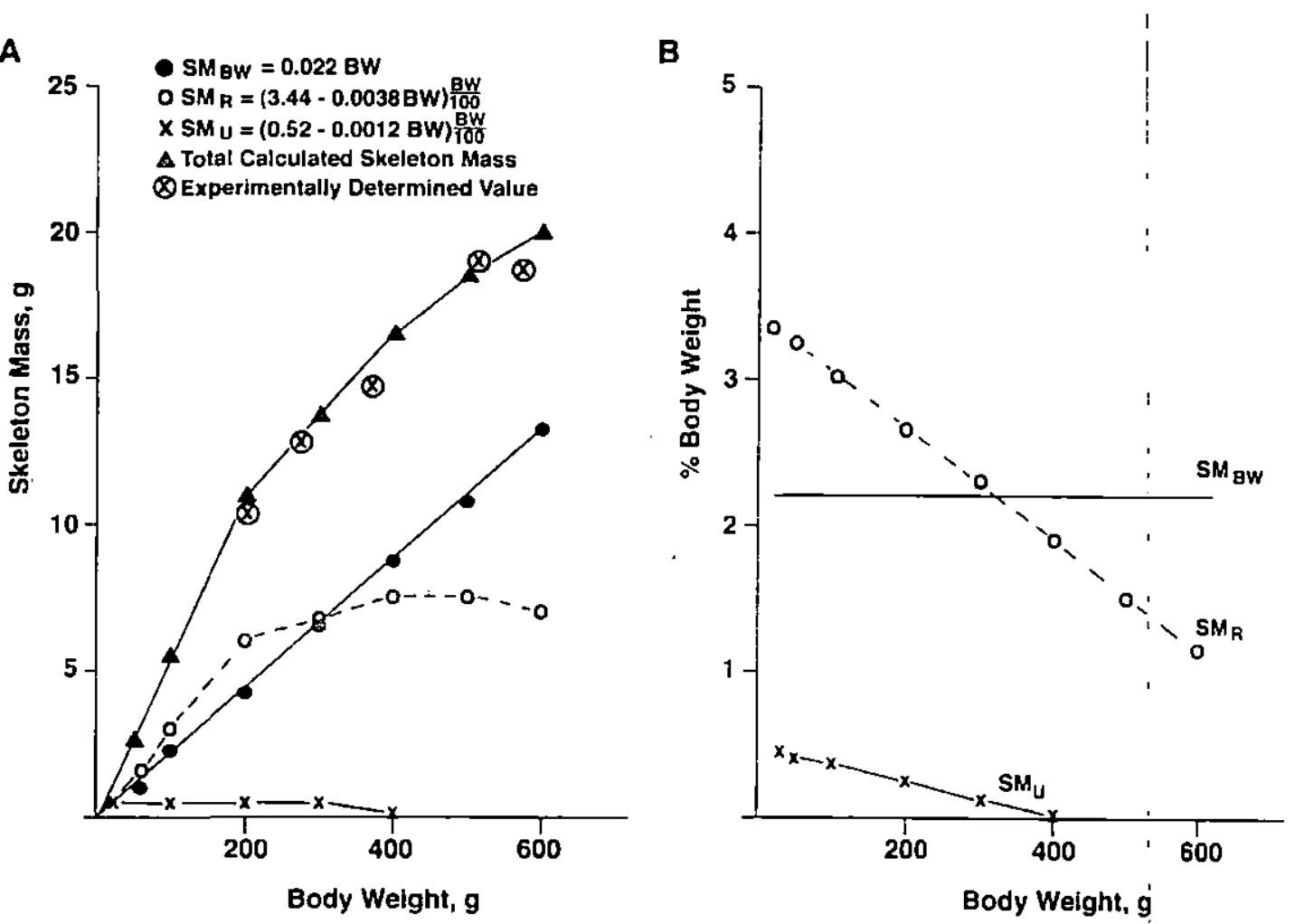

Fig. 5. Values calculated for the different components of skeletal mass (A) indicating their percentage of body weight $(B)$ at a body weight range of $0 \mathrm{~g}$ to $600 \mathrm{~g}$. Components calculated include $S_{B} M_{B}$ the portion of skeleton mass directly correlated with increasing body weight: $\mathrm{SM}_{R}$, the portion skeleton mass developed independently of body weight and directly correlated with bone resorptive activity. and: $\mathrm{SM}_{\mathrm{l}}$ a small component of skeleton mass not directly correlated with either body weight or resorptive activity. Total skeleton mass represents the sum of the individual components with the calculated values $(\boldsymbol{\Lambda})$ compared to experimentally derived data $(\otimes)$ taken from DeMoss and Wright. 1998. 
modeling activity in which bone formation or resorption at a given site may continue uninterrupted for extended intervals, leading to net gains or losses in bone mass $(23,24)$. By comparison, bone metabolic activity in the older rat was confined almost exclusively to remodeling in which bone formation and resorption are tightly coupled, both spatially and temporally $(23,24)$. These studies underline the need for a means to separate bone formation into mechanically and metabolically determined components which may differ in proportion between mature and rapidly growing animals or could be expected to be affected differently by factors which promote bone formation or loss.

The present results indicate that the skeletal mass present in the rat consists of at least two major components. One component $\left(\mathrm{SM}_{\mathrm{Bw}}\right)$ is highly correlated with increasing body weight and may be calculated at approximately $2.2 \%$ of body weight. We suggest that this component reflects mechanically active bone formed and maintained in direct response to structural support demands that is mediated by mechanical strain. A second component $\left(\mathrm{SM}_{R}\right)$ independent of body weight, is inversely correlated with skeletal development and increases with age. This component is highly correlated with bone resorption in mature 24-week-old rats in which remodeling predominates and resorption would be expected to be highly correlated with bone formation. We suggest that this component of skeletal mass is determined or is influenced by factors other than mechanical strain.

In summary, the model presented here enables the calculation of separate components of skeletal mass (Fig. 5A) in presumably normal rats of different, genetically determined body weights at 24 weeks of age. The results indicate that only a portion of skeletal mass $\left(S_{B W}\right)$ increases in direct proportion to increased body weight. A second component is independent of body weight within the weight range of animals studied. In consequence, this component is proportionately more highly developed in small versus large animals (Fig. 5B). We propose that these components represent mechanically and metabolically driven bone formation, respectively. The model may provide significant advantages as a method for evaluating different components of the skeleton in the whole animal compared to assessments among individual bones which may show metabolic and mechanical heterogeneity.

\section{References}

1. DeMoss DL and Wright GL. Sex and strain differencess in whole skeletal development in the rat. Calcif. Tiss. Int. 1998:62:153-157.

2. DeMoss DL and Wright GL. Analysis of whole skeleton 3 H-tetracycline loss as a measure of bone resorption in maturing rats. Calcif. Tiss. Int. 1997:61:412-417.

3. Frost HM. The mechanostat: a proposed pathogenic mechanism of osteoporoses and the bone mass effects of mechanical and nonmechanical agents. Bone and Min. 1987:2:73-85.

4. Frost, HM. Perspectives: why do long distance runners not have more bone? A vital biomechanical explanation and an estrogen effect. J. Bone Miner. Metab. 1997:15:9-16.

5. Frost, HM. Strain and other mechanical influences on bone strength and maintenance. Current Opinion in Orthopedics. 1997;8:60-70.

6. Frost. HM. Osteoporoses: a rationale for further definitions? Calcif. Tiss. Int. 1998:62:89-94.

7. Frost HM, Feretti JL and Jee WSS. Perspectives: some roles of mechanical usage. muscle strength, and the mechanostat in skeletal physiology, disease, and research. Calcif. Tiss. Int. 1998:62:i-7.

8. Frost HM. On rho, a marrow mediator, and estrogen: Their roles in bone strength and "mass" in human females, osteopenias, and osteoporoses - insights from a new paradigm. J. Bone Miner. Metab. 1998;16:113-123. 
sntinue uninter4). By compar$r$ to remodeling and temporally ation into meproportion be$\mathrm{d}$ differently by

; of at least two ng body weight rat this compoise to structural $\mathrm{SM}_{\mathrm{R}}$ ) indepenincreases with veek-old rats in ghly correlated -mined or is in-

components of :termined body 1 mass $\left(\mathrm{SM}_{\mathrm{BW}}\right)$ is independent this component $\therefore$ 5B). We prosone formation, evaluating difits among indi-

te rat. Calcif. Tiss. of bone resorption one mass effects of echanical explanaent Opinion in Or$\rightarrow 4$. s strength, and the in human females. 16:113-123.
9. Schiessi H, Frost HM and Jee WSS. Estrogen and bone-muscle strength and mass relationships. Bone. 1998;22(1):1-6.

10. Minaire P. Skeletal changes during space flight. Clin. Rheumatol. 1989;8:95-103.

11. Rembaut PC and Goode AW. Skeletal changes during space flight. Lancet. 1985;4:1050-1052.

12. Turner RT, Evans GL and Wakley GK. Spacefight results in depressed cancellous bone formation in rat humeri. Aviation, Space. Environ. Med. 1995;66:770-774.

13. Wronski TJ and Morey CL. Inhibition of cortical and trabecular bone formation in the long bones of immobilized monkeys. Clin. Orthop. Rel. Res. 1983;181:269-276.

14. Cann CE and Adachi RR. Bone resorption and mineral excretion in rats during spaceflight. Am. J. Physiol. 1983;244:R327-R331.

15. Weinreb M, Rodan GA and Thompson DD. Osteopenia in the immobilized rat hind limb is associated with increased bone resorption and decreased bone formation. Bone. 1989;10:187-194.

16. Bourrin S, Palle S, Pupier R, Vico L, and Alexandre C. Effect of physical training on bone adaptation in three zones of the rat tibia. J. Bone Miner. Res. 1995;10:1745-1752.

17. Hillam RA and Skerry TM. Inhibition of bone resorption and stimulation of formation by mechanical loading of the modeling rat ulna in vivo. J. Bone Miner. Res. 1995;10:683-689.

18. Rubin CT and Lanyon LE. Regulation of bone formation by applied dynamic loads. J. Bone Joint Surg. Am. 1984:66(A):397-402.

19. Rodan GA. Bone mass homeostasis and bisphosphonate action. Bone. 1997:20(1):1-4.

20. Enlow DH. Function of the Haversian system. Am. J. Anat. 1962:1 10:269-306,

21. Bouvier M and Hylander WL. The mechanical or metabolic function of secondary osteonal bone in the monkey Macaca fascicularis. Arch. Oral Biol. 1996:41(10):94 I-950.

22. Erben RG. Trabecular and endocortical bone surfaces in the ralt: modeling or remodeling?, Anat. Rec. 1996;246:39-46.

23. Frost HM. Intermediary Organization of the Skeleton. Boca Ratan. Fla. CRC Press, 1986.

24. Parfitt AM. The cellular basis of bone remodeling: the quantum concept reexamined in light of recent advances in the cell biology of bone. Calcif. Tiss. Int. 1984;36:S37-S45. 